SUL MOTO SPONTANEO DI UN SOLIDO DI RIVOLUZIONE, VINCOLATO PER UN PUNTO DELL' ASSE AD UN GERCHIO FISSO.

Memoria $1^{a}$ di E. DANIELE

$\$ 4^{0}$ : Moti stazionarii del sistema.

15. Se noi assumiamo come coordinata l'angolo $\chi$ in luogo di w, e quindi, fra le caratteristiche, la derivata $\chi^{\prime}$ in luogo di $\omega^{\prime}$, l'espressione (5) della forza viva fa vedere, poichè mancano le forze applicate, che il sistema possiede due coopdinate cicliche, cioè $\psi$ e $\phi$; i due integrali lineari ( 6$)$ e (7) sarebbero appuntó quelli che provengono da queste due coordinate, cioè non sono altro che le due equazioni

$$
\frac{\partial T}{\partial \varphi^{\prime}}=\text { cost. }, \quad \frac{\partial T}{\partial \psi^{\prime}}=\text { cost. }
$$

Si potrà quindi dedurre da queste due coordinate cicliche una classe di moti stazionarii del sistema, senza alcuna integrazione, mediante la nota regola di Routh. Basterà eguagliare a costanti $\psi^{\prime}$ e $\Phi^{\prime}$ ed annullare $\chi^{\prime}$ e $\Phi^{\prime}$; $i$ valori delle costanti $\psi^{\prime} \phi^{\prime} \chi^{9}$, che corrispondono ad un moto stazionario, sono legati dalle due equazioni

$$
\frac{\partial \mathrm{T}}{\partial \chi}=0, \quad \frac{\partial \mathrm{T}}{\mathrm{d} g}=0
$$

nelle quali si ponga $\chi^{\prime}=\Im^{\prime}=0$. Le (15). scritte distesamente, e ritemendo che $\psi^{\prime}$ è uguale ad $\omega^{\prime}$, diventano:

$$
\text { (15') }\left\{\begin{array}{c}
E R \omega^{\prime 2} \operatorname{sen} \vartheta \cos \chi=0 \\
\left\{(A-C) \operatorname{sen} \vartheta \cos 9 \cdot \omega^{\prime}-C \varphi^{\prime} \cos 9+M R E \omega^{\prime} \cos \vartheta \operatorname{sen} \chi\right\} \omega^{\prime}=0,
\end{array}\right.
$$


e coincidono colle equazioni del sistema ( $\left.A^{\prime}\right)$, che definiscono i moti precessionali, quando vi si ponga $\chi=$ cost. I moti stazionarii del sistema, provenienti dagli integrali (6) e (7), sono dunque $i$ moti precessionali contenuti nella tab. (C), purchè si aggiunga sempre la condizione $\psi^{\prime}=\omega^{\prime}$.

16. Con questo non è però esaurita la questione dei nostri moti stazionarii, Le condizioni scritte nella prima colonna della tåb. (C), che si possono assumere a definizione dei diversi moti stazionarii, non consistono tatte in relazione fra le coordinate e le velocità: i movimenti, cioè, $\mathrm{E}=0$ e $\mathrm{R}=0$ si ottengono non già imponendo qualche nuova condizione alle $\vartheta, \chi, \Phi^{\prime}, \psi^{\prime}$, ma specializzando il valore di un parametro che è in relazione colla natura geometrica del sistema. In altre parole, quelli ora detti sono da considerarsi non tanto come movimenti particolari del sisterna quale noi l'abbiamo definito in generale, quanto piuttosto come movimenti, più o meno particolari, di un sistema che è, in fondo, diverso da quello generico. Se adunque noi vogliamo essere sicuri che l'analisi di questi movimenti riesca completa e nel medesino tempo non porti con sè degli elementi estranej ai movimenti stessi, dovremo ripetere, in loro riguardo, la ricerca della stazionarietà.

Il caso $R=0$ è subito esaurito. Difatti la rotazione spontanea di un solido di rivoluzione intorno ad un punto qualunque del suo asse (che è una precessione regolare) è senza dubbio stazionaria. L'angolo $\chi$ non vi figura per nulla, e quindi il nostro moto precessionale 2), senza bisogno di altre restrizioni. è stazionario.

Per $E=0$ l'espressione (5) della forza viva si riduce in modo da mettere in vista tre coordinate cicliche, ciò̀ $\omega \psi \phi$, e perciò si ha un terzo integrale primo lineare oltre a (6) e (7): questo integrale è

$$
\frac{\partial T}{\partial \omega^{\prime}}=M R^{2} \omega^{\prime}=\text { cost. }
$$

I moti stazionarii più generali provenienti dalla presenza delle coordinate cicliche si ottengono dunque eguagliando a costanti le velocită $\psi^{\prime} \Phi^{\prime} \omega^{\prime}$ e la coordinata 9 ; fra queste costanti 
deve poi passare una relazione, che è la $\frac{\partial \mathrm{T}}{\partial \vartheta}=0$, ossia la $\left(8^{\prime}\right)$.

Se osserviamo che, in luogo di $\omega^{\prime}=$ cost., si potrebbe anche scrivere $\chi^{\prime}=$ cost., vediamo che il movimento del solido per $\mathrm{E}=0$ è stazionario tulte le volte che è un moto precessionale, senza che occorra aggiungere la condizione $\chi=$ cost.

Il risultato trovato alla fine del $\mathrm{n} .15$, che esigeva sempre quest'ultima condizione, è dovuto al fatto che noi includevamo i casi $\mathrm{E}=0$ e $\mathrm{R}=0$ nel caso generale, in cui si hanno due sole coordinate cicliche, e l'angolo $\chi$ è un parametro essenziale. Si vede, del resto, immediatamente quali siano i moti stazionarii particolari che si ottengono per $\mathrm{E}=0$ astraendo da una coordinata ciclica. Ad es., tenendo conto delle sole coordinate cicliche $\psi$ e $\phi$ ed assunto l'angolo $\omega$ come coordinata, ví sarà da porıe, oltre a $\psi^{\prime}$ e $\varphi^{\prime}$ eguali a costanti e $g^{\prime}=0$, anche $\omega^{\prime}=0$ : si trova, ciò̀, il caso in cui il baricentro rimane fisso. Sostituendo alla coordinata $\omega$ l'altra $\chi=\psi-\omega$, che pure risulta ciclica, in luogo di $\omega^{\prime}=0$ vi sará da porre $\chi^{\prime}=0$, e si ritrova così il caso del n. 15 .

Nell'ipotesi che il punto vincolato $\mathrm{P}$ sia fisso $(\mathrm{R}=0)$, l'angolo $\chi$ è un elemento completamente estraneo al moto in sè e per sè considerato; si comprende quindi come, trattando questo caso a parte, ogni condizione relativa a quell' angolo venga a mancare.

Aggiungeremo ancora che un' analoga osservazione si può fare in riguardo al movimento sen $\vartheta=0$, il quale (v. nota al n. 11) dipende da due sole coordinate, $\omega$ e $\lambda=\psi+\phi$, entrambe cicliche, e quindi è stazionario in ogni caso.

Concludendo, possiamo affermare che $i$ moti stazionari del sistema forniti dalla regola di Routh sono esattamente $i$ moti precessionali del $\$$ precedente, a condizione che si lenga conto ogni volta del massimo numero di coordinate cicliche che il sistema presenta, e non s'introducano elementi a questo estranei.

17. La ricerca dei moti stazionarii si può compiere anche senza trasformare la forza viva in modo da avere due coordinate cicliche, ma assumendo a coordinate $\psi \phi \uparrow \chi$ ed a caratte- 
ristiche $\psi^{\prime} \emptyset^{\prime} S^{\prime} \omega^{\prime}$. Basta perciò applicare la regola che dá il prof. Levi-Civita nella sua Nota giá citata Sui moti stazionarii dei sistemi olonomi, dove si suppone che lo stato di movimento del sistema venga rapplesentato con parametri di specie qualunque. Gli integrali (o relazioni invarianti) da cui i moti stazionarii vanno dedotti, sono nel caso nostro gli integrali (6) e (7), i quali sono evidentemente in involuzione, secondo che è richiesto dal metodo del Levi-Civita. I r'isultati a cui si perviene per questa via sono ancora, com'è naturale, identici a quelli già trovati.

$\dot{E}$ inutile che ci fermiamo oltre su questo metodo, la ricerca dei nostri moti stazionarii essendo singolarmente facilitata dalla possibilitá di mettere in evidenza le due coordinate cicliche $\psi$ e $\phi$, e quindi dalla possibilità di applicare senz' al. tro il metodo di Routh.

\section{$\S 5^{0}$ : Esame della stabilità dei moti precedenti.}

18. É noto come per i moti stazionarii la questione della stabilitá si possa decidere nel modo piú ovvio ed esauriente ricorrendo alla sola espressione dell'energia totale. Riferendoci direttamente ai casi che ci interessano, dovremo prendere in considerazione gli integrali primi lineari che noi conosciamo (tutti od una parte), ed eliminare dalla forza viva, col loro mezzo, un ugual numero di caratteristiche; diciamo $\mathbf{T}$ ció che diventa $T$ dopo l'eliminazione: secondochè, per i moti che noi esaminiamo, $\mathbf{T} \dot{e}$, oppure no, un massimo od un minimo effettivo, i moti stessi saranno stabili oppure instabili (teorema di Dirichlet-Liapounoff). Naturalmente si tratterạ di una stabilitá o instabilitá relativa: noi considereremo cioè gli integrali del morimento che ci hanno servito per formare la funzione $\mathbf{T}$, e intenderemo di verificare se il moto in questione è stabile o instabile in confronto con quelli che da esso si deducono in seguito a perturbazioni le quali lasciano inalterate le costanti arbitrarie che entrano in quegli integrali; in altri termini, se noi imaginiamo di sostituire, mediante questi integrali, le costanti d'integrazione ad altrettante caratteristiche, le supposte perturbazioni potranno alterare tutti gli altri parametri 
che individuano lo stato di movimento del sistema, all'infuori di quelle costanti.

Ora, per questi moti il $d \mathbf{T}$ è nullo identicamente, e il $d^{2} \mathbf{T}$ è, in generale, una forma quadıatica nei differenziali dei parametri (di posizione e di velocitá) che non. furono eliminati : noi siamo quindi condotti a verificare se, in corrispondenza a quei movimenti, il $d^{2} \mathbf{T}$ si riduca, oppure no, ad una forma definita.

19. In base al metodo ora ricordato ci proponiamo di studiare la stabilità dei moti stazionarii che provengono dagli integrali (6) e (7), cioè dei movimenti della tab. (C). Fra questi movimenti noi escluderemo però quello che corrisponde a supporre il punto $\mathrm{P}$ coincidente col baricentro $(\mathrm{E}=0)$, il cui studio non presenta alcuna difficoltà dopo la discussione degli altri casi nei quali $P$ è un punto generico dell'asse del solido.

Circa i moti rimanenti andrà osservato che quello in cui il punto $\mathrm{P}$ è fisso [il 2) della tab. (C)] non si potrá più carat. terizzare indifferentemente colle condizioni $R=0$ opp. $\omega^{\prime}=0$. Difatti le due condizioni corrispondono, come s'è osservato al n. 1l, a due modi diversi di concepire il movimento stesso come caso limite del moto generale del solido. Questa diversità di concezione, di nessun conto fintantochè ogni moto vien considerato in sè e per sè, assume invece una grande importanza nella questione della stabilità, non potendosi parlare di moto stabile od instabile senza pensare a tutta una classe di movimenti alla quale il moto considerato appartiene come elemento limite. Ora appunto, la classe dei moti (perturbati) coi quali si paragona il movimento $\omega^{\prime}=0$ per studiarne la stabilità, è ben diver'sa da quella dei movimenti con cui va confrontato il moto $R=0$. Ed invero, siccome l'eguaglianza $R=0$ non esprime una relazione fra le coordinate e la velocità, ogni perturbazione del moto corrispondente lascierà intatta l'eguaglianza stessa, e quindi il moto originario andrà confrontato con altri nei quali il punto $\mathrm{P}$ continua a rimanere fisso; per contro, le perturbazioni del moto stesso, nel quale la quiete del punto $P$ venga rappresentata da $\omega^{\prime}=0$, avranno evidentemente per effetto, fra l'altro, di mettere in moto il punto $P$. 
Ora la stabilitá del movimento, concepita sotto il primo aspeito, è troppo notoria perchè sia il caso di occuparcene; rimane invece da studiare la stabilità dal secondo punto di vista.

Bisognerá dunque pensare, insieme al solido che ruota intorno al punto $\mathrm{P}$, il cerchio su cui verra messo in movimento il punto $\mathrm{P}$ in seguito alla perturbazione. Il suo centro sarà l'origine o degli assi $\infty y z$, il. suo piano determinerà la linea dei nodi come intersezione col piano perpendicolare in $P$ all'asse del solido: cosi compare in forma ben determinata l'angolo $\chi$, che sarebbe privo di significato considerando il moto $\omega^{\prime}=0$ isolatamente; e poichè noi vogliamo ora concepire questo moto come caso limite del moto generale del sistema, dovremo attribuirgli la proprietå $\chi=$ cost. che spetta ai moti stazionarii tutte le volte che si considerano come provenienti dagli integrali (6) e (7) (Cfr. n. 15). Da $\chi=$ cost. e $\omega^{\prime}=0$ segue allora $\psi^{\prime}=0$, e la precessione, di cui dobbiamo esaminare la stabilita, degenera quindi in una rotazione del solido intorno a'l suo asse con velocitå costante $\varphi^{\prime}$.

20. Avremo dunque da occuparci di tre movimenti stazionarii, definiti rispettivamente dalle condizioni.

$$
\omega^{\prime}=0, \quad \cos \chi=0, \quad \operatorname{sen} 9=0,
$$

alle quali bisognerà aggiungere la seconda (15'); siccome però quest'ultima è soddisfatta identicamente per $\omega^{\prime}=0$, cosi non vi sarà da tenerne conto che negli altri due movimenti, e si scriverà, tralasciando nei due membri un fattore w':

$$
\text { MER } \omega^{\prime} \cos \vartheta \operatorname{sen} \chi=\left\{(\mathrm{C}-\mathrm{A}) \omega^{\prime} \cos 9+\mathrm{C} \varphi^{\prime}\right\} \operatorname{sen} 9 .
$$

S'è già detto che una perturbazione del moto $\omega^{\prime}=0$ lancia il punto $\mathrm{P}$, in origine fisso, lungo un cerchio; questo cer. chio, aggiungiamo ora, potrà avere un raggio qualunque, e la velocita, con cui $\mathrm{P}$ viene lanciato, è piccolissima; la pertur. bazione inoltre imprimerà all' asse del solido, inizialmente in quiete, un movimento lungo una rigata generalmente gobba e con linea di gola di dimensioni finite. 
Possiamo anche vedere in che cosa consista una perturbazione del moto $\cos \chi=0$.

L'asse del solido, dapprima in un piano coll'asse $z$, diventi sghembo con questo, staccandosene però per una distanza piccolissima; allora il punto $\mathrm{Q}$ dell'asse $\zeta$, in origine fisso su $z$, vien messo in movimento, descrivendo intorno alla primitiva posizione una curva che ha, almeno all' inizio del moto perturbato, dimensioni piccolissime; della stessa natura sarà la linea di gola della rigata descritta dall'asse $\zeta$ in luogo dell'originario cono di rotazione, mentre il punto $\mathrm{P}$ percorrerà sempre il medesimo cerchio di prima.

Quanto al terzo movimento ( $\operatorname{sen} \vartheta=0$ ), esso verrà perturbato in modo che l'asse $\zeta$ cessi di essere parallelo a $z$. D' altronde la (17), che serve a determinare ulteriormente il moto stazionario, si riduce ora a

$$
\mathrm{E} \omega^{\prime} \operatorname{sen} \chi=0 \text {, }
$$

e poichè all’angolo $\chi$ si può assegnare il valore che si vuole (quando s' intenda di non porre alla perturbazione alcuna re. strizione, oltre a quella di lasciare inalterate le costanti $r$ e $K$ negli integrali (6) e (7) ), così la condizione sen $9=0$ s'accompagna necessariamente o con $\mathrm{E}=0$ opp. con $\omega^{\prime}=0$.

Il porre $\mathrm{E}=0$ significa che la supposta perturbazione deve deviare l'asse $\zeta$ dalla direzione originaria facendolo rotare intorno al baricentro; cioè, dopo la perturbazione il baricentro sarà l'unico punto dell' asse $\zeta$ che continui a descrivere la stessa traiettoria di prima.

Per $\omega^{\prime}=0$ si ricade nel caso del punto $\mathrm{P}$ fisso: la perturbazione potrà mettere in movimento un punto qualunque $\mathrm{P}$ dell'sse $\zeta$ su un cerchio il cui piano sia perpendicolare a $\zeta$, ed in pari tempo deviare la direzione di quest'asse intorno al punto $\mathrm{P}$.

Siccome abbiamo giá detto di non volerci occupare del caso $\mathbf{E}=0$, e poichè, come si vedrà, le conclusioni a cui giungeremo riguardo alla stabilità del moto $\omega^{\prime}=0$ valgono qualunque sia il valore di $\vartheta$ (anche per $\vartheta=0$ ), vuol dire che non avremo da considerare esplicitamente il moto stazionario 3) della tab. (C): non rimangono quindi che i due moti $\omega^{\prime}=0$ e $\cos \chi=0$. 
21. Risolvendo le (6) e (7) rispetto a $\psi^{\prime}$ e $\Phi^{\prime}$ si trova:

$$
\psi^{\prime}=\frac{\pi-A r \cos 9-M R U \omega^{\prime}+M E R 9^{\prime} \cos 9 \cos \chi}{V \operatorname{sen} \vartheta}
$$

$\varphi^{\prime}=\frac{(A+M E R \operatorname{sen} 9 \operatorname{sen} \chi) r-K \cos 9+M R \cos \vartheta\left(U \omega^{\prime}-E \vartheta^{\prime} \cos 9 \cos \chi\right)}{V \operatorname{sen} 9}$,

avendo posto

$$
U=R+E \operatorname{sen} i \operatorname{sen} \chi, \quad V=A \operatorname{sen} \gamma+M E R \operatorname{sen} \chi .
$$

Si tratterà adesso di sostituire queste, espressioni di $\psi^{\prime}$ e $\phi^{\prime}$ in T, e calcolare le derivate parziali della funzione $\left.\mathbf{T}^{\prime} \omega^{\prime} \boldsymbol{S}^{\prime} \chi^{9}\right)$ che si ottiene. Bisognerà poi specialízzare queste derivate pei movimenti che ci interessano, e quindi supporre anzitutto $S^{\prime}=0$ e $\chi^{\prime}=\psi^{\prime}-\omega^{\prime}=0$. Facendo uso della (7) e della (17), le quali, eliminata $\Phi^{\prime}$ mediante la (6), prendono risp. la forma

$$
\left\{\begin{array}{c}
(\mathrm{V} \operatorname{sen} \theta+\mathrm{MRU}) \omega^{\prime}+\mathrm{A} r \cos \theta-\mathrm{K}=0 \\
\mathrm{~V} \omega^{\prime}-\mathrm{C} r \operatorname{tg} \theta=0,
\end{array}\right.
$$

si verifica facilmente che le derivate prime di $\mathbf{T}$ son nulle tutte le volte che sia soddisfatta una qualunque delle (16).

Si calcoleranno in seguito le derivate seconde di $\mathbf{T}$, le quali acquisteranno espressioni differenti in corrispondenza ai due movimenti $\omega^{\prime}=0$ e $\cos \chi=0$.

Cominciando dal primo, si trova che son nulle tutte le derivate seconde di $T$ nelle quali figura almeno una derivazione rispetto a $\chi$, e le altre derivate hanno le espressioni seguenti:

(19)

$$
\begin{aligned}
& \left(\omega^{\prime} \omega^{\prime}\right)=\frac{M R^{2}}{V^{2}}\left(A-M E^{2} \operatorname{sen}^{2} \chi\right)(V \operatorname{sen} \vartheta+M R U) \\
& \left(\mathcal{I}^{\prime} 9^{\prime}\right)=A\left(1+\frac{M^{2} E^{3} R^{2} \cos ^{2} 9 \cos ^{2} \chi}{V^{2}}\right) \\
& \left\{\begin{array}{l}
\left(\omega^{\prime} 9^{\prime}\right)=-\frac{\mathrm{AMER} \cos 9 \cos \chi}{\mathrm{V}^{2}}(\mathrm{~V} \operatorname{sen} \vartheta+\mathrm{MRU}) \\
\left(\omega^{\prime} \vartheta\right)=-\frac{\mathrm{CMR}^{2} r \operatorname{sen} \vartheta}{\mathrm{V}^{3}}\left(\mathrm{~A}-\mathrm{ME}^{2} \operatorname{sen}^{2} \chi\right)
\end{array}\right. \\
& \left(\vartheta^{\prime} 9\right)=\frac{A C M E R r \operatorname{sen} 9 \cos 9 \cos \chi}{V^{2}},(9 \vartheta)=\frac{A C^{2} r^{2} \operatorname{sen}^{2} \vartheta}{V^{2}} .
\end{aligned}
$$


Per brevità abbiamo scritto $\left(\omega^{\prime} \omega^{\prime}\right),\left(\omega^{\prime} S^{\prime}\right), \ldots$. in luogo di $\frac{\partial^{2} T}{\partial \omega^{12}}, \frac{\partial^{2} T}{\partial \omega^{1} d g^{1}} \ldots$

Mostreremo ora che per il movimento in questione la forma $a^{z} \mathbf{T}$ è definita positiva.

Se $\Delta$ è il discriminante della forma, ciò̀ se poniamo

$$
\Delta=\left|\begin{array}{lll}
\left(\omega^{\prime} \omega^{\prime}\right) & \left(\omega^{\prime} \vartheta^{\prime}\right) & \left(\omega^{\prime} \vartheta\right) \\
\left(\vartheta^{\prime} \omega^{\prime}\right) & \left.\left(\vartheta^{\prime}\right)^{\prime}\right) & \left(\vartheta^{\prime} \vartheta\right) \\
\left(\vartheta \omega^{\prime}\right) & \left(\vartheta \vartheta^{\prime}\right) & (\vartheta \vartheta)
\end{array}\right|,
$$

faremo vedere che si hanno le seguenti disuguaglianze:

$$
\left(\omega^{\prime} \omega^{\prime}\right)>0, \quad\left(\omega^{\prime} \omega^{\prime}\right)\left(S^{\prime} \hat{J}^{\prime}\right)-\left(\omega^{\prime} g^{\prime}\right)^{2}>0, \quad \Delta>0,
$$

i cui primi membri non sono altro che $\mathrm{i}$ minori principali di $\Delta$, partendo dal primo elemento della diagonale principale e andando fino a $\Delta$ stesso. Per i noti teoremi sulle forme quadritiche il provare quelle disuguaglianze equivale appunto a provare che la forma $d^{2} \mathbf{T}$ è definita positiva.

La prima condizione da verificare è [v. le (19)], astraendo dal fattore positivo $\frac{M R^{2}}{V^{2}}$ :

$$
\left(A-M E^{2} \operatorname{sen}^{2} \chi\right)(V \operatorname{sen} 9+M R U)>0
$$

Ora $\mathrm{A}-\mathrm{ME}^{\mathbf{2}}$ è il momento d'inerzia del solido rispetto ad un asse baricentrico perpendicolare all'asse $\zeta$, per cui è una quantitá positiva, e quindi ̀̀ pure positivo $A-M E^{3} \operatorname{sen}^{2} \chi$. Inoltre l' espressione

$$
V \operatorname{sen} 9+\mathrm{MRU}=\mathrm{A} \operatorname{sen}^{2} 9+2 \mathrm{MER} \operatorname{sen} 9 \operatorname{sen} \chi+\mathrm{MR}^{2},
$$

considerata come polinomio di $2^{0}$ grado in sen 9 , ha per discriminante

$$
M R^{2}\left(A-M E^{2} \operatorname{sen}^{2} \chi\right)
$$


e quindi è positiva qualunque siano $\$$ e $\chi$. Si ha dunque, per $\omega^{\circ}=0$ :

$$
\left(\omega^{\prime} \omega^{\prime}\right)>0
$$

La seconda condizione che vogliamo verificare si esprime attualmente cosi :

$$
\begin{gathered}
\frac{A M R^{2}}{V^{4}}\left[\left(V^{2}-2 M E U V \operatorname{sen} \chi+A M U^{2} ;\left(V^{2}+M^{2} E^{2} R^{2} \cos ^{2} 9 \cos ^{2} \chi\right)\right.\right. \\
\left.-A M E^{2} \cos ^{2} \vartheta \cos ^{2} \chi(V \operatorname{sen} \vartheta+M R U)^{2}\right]>0
\end{gathered}
$$

dopo alcune trasformazioni dell'espressione entro parentesi quadre si viene a dare al primo membro la forma seguente:

$$
\frac{A M R^{2}}{V^{2}}(V \operatorname{sen} \theta+M R U)\left\{A-M E^{2}\left(\cos ^{2} \theta+\operatorname{sen}^{2} \theta \operatorname{sen}^{2} \chi\right)\right\} \text {. }
$$

Si vede ora facilmente che

$$
\cos ^{2} 9+\operatorname{sen}^{2} 9 \operatorname{sen}^{2} \chi
$$

è sempre compreso fra 0 e 1 col variare di $\vartheta$ e $\chi$; ne segue che l'ultimo prodotto scritto ha tutti i suoi fattori positivi.

Veniamo all' ultima $(20)$, cioè $\Delta>0$. Sviluppando $\Delta$ secondo gli elementi della prima orizzontale si ha:

dove

$$
\Delta=\left(\omega^{\prime} \omega^{\prime}\right) \delta_{1}+\left(\omega^{\prime} g^{\prime}\right) \delta_{2}+\left(\omega^{\prime} \vartheta\right) \delta_{\mathrm{s}},
$$

$\delta_{1}=\frac{\mathrm{C}^{2} \mathrm{~A}^{2} r^{2} \operatorname{sen}^{2} \Omega}{\mathrm{V}^{3}}$

$$
\delta_{2}=\frac{C^{2} \mathrm{AMER} r^{2} \operatorname{sen}^{2} 9 \cos 9 \cos \chi}{\mathrm{V}^{2}}
$$

$$
\begin{array}{r}
\delta_{3}=\frac{\mathrm{CAMR}^{2} r \operatorname{sen} \vartheta}{\mathrm{V}^{4}}\left\{\left(\mathrm{~A}-\mathrm{ME}^{2} \operatorname{sen}^{2} \chi\right)\left(\mathrm{V}^{2}+\mathrm{M}^{2} \mathrm{E}^{2} \mathrm{R}^{2} \cos ^{2} 9 \cos ^{2} \chi\right)\right. \\
\left.-\mathrm{AME}^{2} \cos ^{2} 9 \cos ^{2} \chi(\mathrm{V} \operatorname{sen} \vartheta+\mathrm{MRU})\right\}
\end{array}
$$

si ricava quindi facilmente:

$$
\Delta=\frac{\mathrm{C}^{2} \mathrm{AMR}^{2} r^{2} \operatorname{sen}^{2} \vartheta}{\mathrm{V}^{2}}\left\{\mathrm{~A}-\mathrm{ME}^{2}\left(\operatorname{sen}^{2} \chi+\cos ^{2} 9 \cos ^{2} \chi\right)\right\},
$$


e tutti i fattori di questa espressione sono positivi, perchè si ha:

$$
\operatorname{sen}^{2} \chi+\cos ^{2} 9 \cos ^{2} \chi=\cos ^{2} \theta+\operatorname{sen}^{2} \vartheta \operatorname{sen}^{2} \chi .
$$

Per $\omega^{\prime}=0$ il $d^{2} \mathbf{T}$ è dunque una forma definita positiva, e noi possiamo formulare il seguente caso di stabilitá:

Un solido di ricoluzione rispelto ad un asse contenente il baricentro, non soggetto a forze, ruota uniformemente intorno al suo asse; si perturbi il movimento in modo che tw punto qualunque $\mathrm{P}$ dell' asse venga spinto lungo un cer. chio qualunque, ma in modo però che siano sempre scddisfatte le (6) e (7) per gli stessi valori di $r$ e $\mathrm{K}$ : rispetto ad una tale perturbazione il moto originario è stabile.

22. Un dubbio sulla legittimità di questo enunciato potrebbe nascere pensando che il punto $\mathrm{P}$, per quanto riceva inizialmente una velocità piccolissima, potrà trovarsi, dopo un tempo sufficientemente grande, ad una distanza notevolmente grande dalla posizione primitiva; di guisa che il moto, da noi classificato come stabile, non possederebbe tutti i caratteri che noi comunemente comprendiamo nel concetto di stabilità. Ma il dubbio accennato dovrebbe allora cadere anche sul moto ret. tilineo uniforme di un punto, il quale muovendosi, in seguito ad una perturbazione, di un moto analogo su una retta formante colla prima un angolo sia pure piccolissimo, può venirsi a trovare, dopo un tempo molto grande, ad una distanza comunque grande dalla posizione che il punto avrebbe nel moto originario; eppure nessuno oserebbe chiamare instabile il moto rettilineo uniforme di un punto. Si capisce come l'attributo di stabile o instabile dato ad un movimento dipenda dalla definizione che noi assumiamo di stabilità ; e come possa occor. rere di allargare, nella definizione, in seguito ad una opportuna critica, il concetto comune di stabilita per non incorrere nel pericolo di non riuscire più a trovare nessun moto al quale competa la denominazione di stabile. Si legga, a questo proposito, la bellissima discussione che i sigg. Klein e Sommerfeld fanno del concetto di stabilità nel Cap. V, $\$ 6$ della più volte citata Theorie des Kreisels. 
23. Passiamo al moto $\cos \chi=0$. Le derivate seconde di $\mathrm{T}$ ora diventano:

$$
\begin{aligned}
& \left(\omega^{\prime} \omega^{\prime}\right)=\frac{M R^{2}}{V^{2}}\left(A-M E^{2}\right)(V \operatorname{sen} \vartheta+M R U) \\
& \left.\left(\mathcal{G}^{\prime}\right)^{\prime}\right)=\mathrm{A}, \quad\left(\omega^{\prime} \mathcal{S}^{\prime}\right)=\left(\omega^{\prime} \chi\right)=0 \\
& \left(\omega^{\prime} 9\right)=\frac{M R^{2} \omega^{\prime} \cos \vartheta}{V}\left(A-\mathrm{ME}^{2}\right) \\
& \left(9^{\prime} \chi\right)=\left(g^{\prime} 9\right)=\left(\chi^{9}\right)=0 \\
& (\chi x)= \pm M E R \omega^{\prime 2} \operatorname{sen} s, \quad(\vartheta y)=\frac{V_{\omega}^{\prime 2}}{s e 11},
\end{aligned}
$$

ed il discriminante di $d^{2} \mathbf{T}$ diventa:

$$
\Delta=\left|\begin{array}{cccc}
\left(\omega^{\prime} \omega^{\prime}\right) & 0 & 0 & \left(\omega^{\prime} \vartheta\right) \\
0 & \left(\vartheta^{\prime} \vartheta^{\prime}\right) & 0 & 0 \\
0 & 0 & (\chi \chi) & 0 \\
\left(\vartheta \omega^{\prime}\right) & 0 & 0 & (\vartheta \vartheta)
\end{array}\right|
$$

Bisognerà ora distinguere $i$ due casi : $\operatorname{sen} \chi=+1$ oppure $\operatorname{sen} \chi=-1$.

Supposto, in primo luogo, sen $\chi=+1$, ci proponiamo di dimostrare che sono quantità positire tutti i minori principali di $\Delta$; vogliamo ciò̀ dimostrare le seguenti disuguaglianze:

$$
\text { (21) }\left\{\begin{array}{l}
\left(\omega^{\prime} \omega^{\prime}\right)>0 \\
\left(g^{\prime} \xi^{\prime}\right)=\mathrm{A}>0 \\
(\chi \chi)=\mathrm{MER} \omega^{\prime 2} \operatorname{sen} \Omega>0 \\
\Delta>0 .
\end{array}\right.
$$

Quanto alla prima è certo soddisfatta, perchè s'è visto nella discussione del moto $\omega^{\prime}=0$ che si ha $\left(\omega^{\prime} \omega^{\prime}\right)>0$ qualunque siano 9 e $\chi$.

La seconda poi è soddisfatta senz'altro. Circa la terza dobbiamo osservare che, incontrandosi gli assi $z$ e $\zeta$, è da escludersi $\operatorname{sen} 9=0$; inoltre $\vartheta$, per la sua stessa definizione ed in quanto si considera un determinato moto stazionario (in cui $\vartheta$ ha un valore costante) è compreso fra 0 e $\pi$, onde sen $2>0$ : ne segue che la terza è verificata essa pure. 
Rimane da considerare la disuguaglianza $\Delta>0$. Si ha:

$$
\left.\Delta=\left(\Im^{\prime} \vartheta^{\prime}\right)(\chi \chi)\left[\omega^{\prime} \omega^{\prime}\right)(\Im \vartheta)-\left(\omega^{\prime} \Im\right)^{2}\right],
$$

e poichè i due primi fattori sono positivi, basterà che verifichiamo il segno del terzo. Ora si vede facilmente che a quel fattore si puó dare la forma seguente:

$$
\begin{aligned}
\frac{A-M E^{2}}{(A \operatorname{sen} 2+M E R) \operatorname{sen} I}\left[\left\{A^{2}+\left(A-M E^{2}\right) M R^{2}\right\} \operatorname{sen}^{3} I+\right. \\
\left.+3 A M E R \operatorname{sen}^{2} I+3 M^{2} E^{2} R^{2} \text { sen } 9+M^{2} E R^{3}\right]
\end{aligned}
$$

per le osservazioni giả fatte sui segni di $A-M E^{3}$ e di sen 9 questa espressione è positiva.

Risultano quindi soddisfatte le (21), onde concludiamo che per il moto stazionario corrispondente a sen $\chi=+1$ la forma quadratica $d^{2} \mathbf{T}$ è definita positiva.

Facciamo invece $\operatorname{sen} \chi=-1$. Abbiamo ancora

$$
\left(\omega^{\prime} \omega^{\prime}\right)>0,\left(S^{\prime} S^{\prime}\right)>0 \text {, }
$$

ma per contro

$$
(\chi \chi)=-\mathrm{MER} \omega^{\prime 2} \operatorname{sen} \mathrm{S}<0,
$$

mentre in $\Delta$ ri è, oltre al fattore negativo $(\chi \chi)$, il divisore A sen $\Im-M E R$ che puó prendere il segno +0 il segno a seconda dei valori di $T$ e delle costanti A, M, E, R. In conseguenza, per sen $\chi=-1$ la forma $d^{2} T$ non è definita.

Ricordando l'osservazione fatta al n. 13, che si ha sen $\chi= \pm 1$ secondochè il punto $P$ cade internamente od esternamente al seymento finito $Q G$, possiamo enunciare il risultato che segue: Un solido, simmetrico rispello ad un asse bavicentrico $\zeta$ di cui un punto $\mathrm{P} \dot{e}$ assoggettalo a muoversi sopra un cerchio fisso, descrive intorno ad un

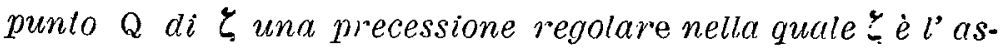
se precessionale mohile; si dia al movimento una perturbazione in modo che a) il punlo $\mathrm{P}$ continui a descrivere il medesimo cerchio che descriveva nel moto miginario, $e-b)$ continuino ad essere snddis/atte le (6) $e$ (7) per gli stessi valori di $r$ e $\mathrm{K}$ a di fronte ad una tale perturbazione 
la primitiva precessione regolave è stabile od instabile secondo che il punto $\mathrm{P}$ cade internamente od esternamente al segmento $\mathrm{Q} G$.

24. S'è detto al $n$. 20 che per sen $\Im=0$ si ricade necessariamente o nel moto $\mathrm{E}=0$ o nel moto $\omega^{\prime}=0$. Quest' ultimo fu trattato esaurientemente al n. 21 ; quanto al primo ci limiteremo ad accennare che il moto stazionario più generale, corrispondente ai tre integrali primi lineari che il sistema ammette, consiste in una rotazione uniforme del punto $G$ intorno ad $O$, unita ad una precessione regolare del solido intorno a $G$ coi due assi precessionali $z$ e $\zeta$, la quale si svolge colla legge espressa dalla $\left(8^{\prime}\right)$. Questo movimento è stabile rispetto a quelle perturbazioni che lasciano inalterate le costanti arbitrarie in quegli integrali, e cioè, tenuto conto del loro significato, che non mutano nè la velocitá di $G$ su $(\gamma)$, nè i momenti, rispetto agli assi $z_{1}$ e $\zeta$, della quantita di moto del solido nella sua rotazione intorno a $\mathrm{G}$. 